\title{
Metodologias ativas em uma capacitação sobre humanização da assistência neonatal: avaliação da qualidade em foco
}

\author{
Alfredo Almeida Pina-Oliveira1, Camila Aparecida Rodrigues Carriel², Anna Maria Chiesa ${ }^{3}$
}

\begin{abstract}
RESUMO
A morbimortalidade perinatal persiste e representa desafio para a garantia da qualidade do cuidado prestado em unidades de terapia intensiva neonatal. Objetivo: descrever e analisar os principais aspectos de uma capacitação sobre a humanização do cuidado no método-canguru com potenciais efeitos na qualidade da assistência. Método: trata-se da análise dos documentos produzidos durante oficinas de reedição baseado em metodologias ativas a partir do referencial donabediano de avaliação da qualidade. Resultados: Foram treinados 26 profissionais da assistência e da gestão hospitalar e foram registradas a redução de infecções hospitalares neonatais e a promoção do cuidado humanizado após 10 meses do término do processo educativo. Conclusão: oficinas de reedição representam estratégia adequada para a melhoria da qualidade do cuidado por meio da valorização dos conhecimentos dos próprios trabalhadores e do contexto no qual se inserem, fortalecendo a perspectiva da educação permanente baseada em metodologias ativas de ensino e de aprendizagem.
\end{abstract}

Descritores: Capacitação em Serviço; Humanização da Assistência; Gestão de Qualidade; Reorganização de Recursos Humanos; Método Canguru.

\section{Active methodologies for teaching humanization of neonatal care: quality assistance assessment in focus}

\begin{abstract}
Perinatal morbidities and deaths remain a challenge in order to achieve improving quality and humanization of healthcare in neonatal intensive care units. Our aim was to describe and analyze an in-service training regarding kangaroo-mother care method focused on quality of care. We have chosen documental analysis from records that had been yielded in this training based on active methodologies teaching and assessed by Donabedian's quality management evaluation. Twenty six care and management professionals were trained. It has been reported decrease of neonatal infections and change on care delivered on humanization principles 10 months after the training. It was presented pedagogical dimensions and their interfaces with human resources management. We conclude that replication training has provided resourceful elements for ongoing improvement of quality of care considering workers' knowledge and their own worksite context, strengthening permanent education grounded on active methodologies of teaching and learning.
\end{abstract}

Descriptors: Inservice Training; Humanization of Assistance; Quality Management; Personnel Turnover; Kangaroo-mother Care Method.

${ }^{1}$ Doutorando em Ciências da Saúde pela Escola de Enfermagem da Universidade de São Paulo (EEUSP), São Paulo, SP, Brasil.

${ }^{2}$ Especialista em Enfermagem Neonatal e Pediátrica pela Universidade Nove de Julho (UNINOVE), São Paulo, SP, Brasil.

${ }^{3}$ Doutora em Saúde Pública pela Universidade de São Paulo (USP), São Paulo, SP, Brasil. 


\section{Introdução}

A mortalidade infantil em países em desenvolvimento, em especial, no cenário brasileiro apresenta queda contínua e significativa. Contudo, a mortalidade e morbidade perinatal ainda persistem e são decorrentes de distúrbios ou circunstâncias superpostas ao curso normal de eventos associados com o nascimento e ajustamento à existência extrauterina ${ }^{1-4}$.

A Unidade de Terapia Intensiva Neonatal (UTIN) configura-se como um espaço de produção de cuidado integral ao recém-nascido (RN) e à família para o controle de eventos adversos e complicações que, muitas vezes, impediam os cuidados maternos e enfatizavam a atuação da equipe de saúde durante a internação neonatal ${ }^{4-6}$.

Para tanto, a integração da equipe de cuidados neonatais com a família do RN de risco torna-se relevante para a evolução do tratamento de maneira sistematizada a fim de garantir o bem estar e segurança do RN no ambiente da UTIN e promover a humanização da assistência prestada a ele e à sua família com foco na gestão da qualidade ${ }^{7-9}$.

A qualidade da assistência requer capacitações em serviço que promovam a humanização e a garantia de cuidados seguros ao RN. A lacuna do conhecimento nessa área ${ }^{1,2}$ denota a necessidade de formação contínua dos profissionais que extrapole modelos pedagógicos tradicionais e adote metodologias ativas que problematizem a formação no ambiente de trabalho.

Por essa razão, o objetivo desse estudo foi descrever e analisar os principais aspectos de uma capacitação sobre a humanização do cuidado no método-canguru com potenciais efeitos na qualidade da assistência.

\section{Material e método}

Trata-se de uma análise documental, descritiva e exploratória, baseada nas recomendações metodológicas propostas na realização de estudos de caso ${ }^{11,12}$. Relacionou-se ao processo educativo organizado em oficinas de reedição, isto é, para a nova formação de profissionais em seus contextos de atuação com a finalidade de difundir o conhecimento sobre a Humanização da Assistência em uma Unidade de Terapia Intensiva Neonatal (UTIN) de um Hospital Geral Estadual.

Justifica-se a escolha dessa instituição por se tratar de referência para gestação de risco no litoral paulista com atendimento regionalizado e administração indireta-autárquica, com oferta de 94 leitos de internação, sendo que 10 são destinados à UTIN.

As oficinas de reedição em foco foram fundamentadas nos princípios da metodologia da problematização no contexto da educação permanente ${ }^{13}$ e na educação baseada em problemas ${ }^{14}$. Optou-se pelas cinco etapas da metodologia da problematização para a estruturação da capacitação em serviço, a saber: observação da realidade, identificação de pontos-chaves, teorização, hipótese para a solução e aplicação à realidade.

A coordenação desse processo educativo foi assumida por duas enfermeiras e uma médica da UTIN da referida instituição e que participaram de um curso de 40 horas de capacitação de tutores em "Atenção humanizada ao recémnascido - método canguru"14,15 realizado em parceria com hospital da capital paulista, no período de agosto de 2010.

Após três meses dessa capacitação de tutores, 26 profissionais foram convocados pela chefia de Enfermagem e divididos em duas turmas com três dias alternados de formação para cada uma das turmas, perfazendo uma carga horária total de seis horas por turma. Participaram integrantes da equipe de enfermagem, médicos, fisioterapeutas, assistentes sociais, coordenadores de serviços técnicos e administrativos e diretor técnico do hospital.

As oficinas de reedição foram realizadas em local próprio da instituição com uso de recursos audiovisuais, material didático, reprografia, elementos para simulação dos cuidados com o RN, lanches para os participantes e transporte de professor convidado. A própria instituição custeou esse processo educativo e autorizou o pagamento de hora extra aos profissionais convocados fora da sua jornada de trabalho habitual.

Os documentos produzidos nessas diferentes etapas representaram o corpus desse estudo, a saber: o plano de implementação de oficinas de reedição após capacitação externa, a ata do encontro com gestores, o projeto de disseminação de conhecimento, o diagnóstico situacional das unidades do hospital, os planos de aulas, o cronograma das atividades, o relatório da supervisão trimestral e o relatório gerencial de implantação do método canguru na UTIN após 10 meses das oficinas de reedição.

Para a caracterização e a análise do conteúdo desse material empírico, adotaram-se o referencial analítico de estudo de caso único holístico de Robert R. Yin ${ }^{11}$ teórico dos sete pilares da qualidade propostos por Avedis Donabedian quais sejam: a eficácia (ciência e tecnologia na produção de melhorias na assistência à saúde), a eficiência (redução de custo da assistência sem diminuir as melhorias obtidas), a efetividade (alcance do melhor estado de saúde baseado nas melhores evidências científicas disponíveis), a otimização (relação custo-benefício na assistência à saúde), a 
aceitabilidade (adequação dos cuidados de saúde às necessidades, desejos e expectativas dos clientes e seus familiares), a legitimidade (concordância entre os costumes, valores éticos, morais, leis e regulamentos da assistência à saúde) e a equidade (justiça e honestidade na distribuição dos serviços de saúde e seus benefícios).

Por se tratar de um estudo em base documental não houve a submissão ao Comitê de Ética em Pesquisa, porém solicitou-se a autorização dos responsáveis pela capacitação e adotaram-se os princípios éticos norteadores de pesquisas em saúde para o relato dessa experiência institucional.

\section{Resultados}

Os documentos das oficinas de reedição forneceram elementos (Figura 1) para potencializar a difusão de novos conhecimentos e abordagens para o cuidado em UTIN com foco na humanização, valorizando a experiência dos próprios educandos/trabalhadores e o emprego de metodologias ativas, tais com a problematização e a aprendizagem baseada em problemas.

Figura 1 - Caracterização das oficinas de reedição sobre humanização do método canguru à luz do referencial donabediano de avaliação de qualidade

\begin{tabular}{|c|c|c|}
\hline Etapa das oficinas de reedição & Estratégias e recursos didáticos & Pilar (es) da qualidade \\
\hline \multirow[t]{3}{*}{$\begin{array}{l}\text { Planejamento dos coordenadores e } \\
\text { atores institucionais relevantes }\end{array}$} & $\begin{array}{c}\text { Diagnóstico situacional das unidades } \\
\text { hospitalares }\end{array}$ & Eficácia e efetividade \\
\hline & $\begin{array}{l}\text { Encontro com gestores e potenciais } \\
\text { participantes }\end{array}$ & $\begin{array}{c}\text { Eficácia, eficiência, otimização, } \\
\text { aceitabilidade legitimidade e equidade }\end{array}$ \\
\hline & $\begin{array}{c}\text { Delineamento das oficinas de reedição } \\
\text { com foco na humanização do método } \\
\text { canguru }\end{array}$ & $\begin{array}{l}\text { Eficácia, eficiência, otimização, } \\
\text { aceitabilidade e equidade }\end{array}$ \\
\hline \multirow[t]{6}{*}{ Execução do processo educativo } & $\begin{array}{c}\text { Recepção e distribuição do material } \\
\text { didático }\end{array}$ & Eficiência e otimização \\
\hline & $\begin{array}{l}\text { Exposição das expectativas e } \\
\text { apresentação dos objetivos de } \\
\text { aprendizagem }\end{array}$ & $\begin{array}{c}\text { Eficácia, efetividade, aceitabilidade e } \\
\text { equidade }\end{array}$ \\
\hline & $\begin{array}{l}\text { Emprego da estrutura e dos recursos } \\
\text { audiovisuais da própria instituição }\end{array}$ & $\begin{array}{c}\text { Eficiência, aceitabilidade, equidade e } \\
\text { otimização }\end{array}$ \\
\hline & $\begin{array}{l}\text { Apresentação da metodologia ativa de } \\
\text { ensino aprendizagem }\end{array}$ & Eficácia e eficiência \\
\hline & $\begin{array}{c}\text { Exposições dialogadas, estudo de caso } \\
\text { e aulas práticas }\end{array}$ & $\begin{array}{l}\text { Eficácia, efetividade, aceitabilidade, } \\
\text { legitimidade e equidade }\end{array}$ \\
\hline & $\begin{array}{l}\text { Metodologias ativas e fechamento do } \\
\text { estudo de caso }\end{array}$ & $\begin{array}{c}\text { Eficácia, eficiência, efetividade, } \\
\text { otimização, aceitabilidade, } \\
\text { legitimidade e equidade }\end{array}$ \\
\hline \multirow[t]{3}{*}{ Avaliação do processo educativo } & Avaliação em grupo & Eficácia, efetividade e aceitabilidade \\
\hline & Auto avaliação dos participantes & Legitimidade e aceitabilidade \\
\hline & $\begin{array}{l}\text { Reprodutibilidade das oficinas de } \\
\text { reedição no contexto do trabalho dos } \\
\text { participantes }\end{array}$ & $\begin{array}{l}\text { Eficácia, eficiência, efetividade, } \\
\text { otimização, aceitabilidade, } \\
\text { legitimidade e equidade }\end{array}$ \\
\hline
\end{tabular}

Cada etapa da problematização foi realizada pelos participantes para a reedição da capacitação de tutores sobre humanização da assistência ao RN em suas unidades de origem, assistenciais ou gerenciais.

A observação da realidade ocorreu com a equipe original (tutores capacitados) e a equipe das oficinas de reedição descritas por meio da documentação analisada. Identificaram-se fragilidades e limites para a prestação de cuidados baseados nas premissas da humanização da assistência em UTIN e levantaram-se dados de infecção hospitalar neonatal.

Os principais pontos-chaves foram: a construção do vínculo empático no método canguru, a superação do cuidado exclusivamente técnico, o alívio e controle da dor no RN, a abordagem familiar, a qualidade da assistência humanizada e a própria metodologia da problematização para futuras replicações em outros contextos e locais de trabalho. 
Valorizaram-se os conhecimentos adquiridos na etapa de teorização entre pequenos grupos da equipe de reedição a fim de produzir hipóteses para a solução viáveis e realistas com base nos recursos humanos, materiais e financeiros da UTIN.

A aplicação do novo conceito à realidade permitiu a construção de alternativas do cuidar em UTIN e ampliar a abordagem com a família do RN. Registrou-se a alta adesão e satisfação dos participantes, o controle da rotatividade entre profissionais de outros setores na UTIN e a redução das taxas de infecção hospitalar neonatal.

As equipes de UTIN registraram melhoria expressiva na redução de casos de infecção neonatal. Dez meses que antecederam as oficinas de reedição, foram realizadas 82 admissões e, após internação, identificaram-se 10 casos de infecção hospitalar (12,19\%). Nos dez meses posteriores, houve 73 admissões e apenas um caso de infecção hospitalar (1,37\%).

Após dez meses do processo educativo analisado, o aumento da rotatividade de trabalhadores com remanejamento de $85 \%$ da equipe treinada representou retrocesso, pois das 69 internações no período ${ }^{11}$, tiveram infecção hospitalar $(15,94 \%)$, indicando que a equipe treinada no processo descrito contribuía para a garantia da qualidade do cuidado.

\section{Discussão}

A formação contínua na vida profissional e o apoio político-institucional representam estratégias para a melhoria da qualidade em saúde ${ }^{7-10}$. Para o referencial donabediano, as oficinas de reedição propiciaram a verificação de indicadores de estrutura16, sem desconsiderar as interfaces para a produção de indicadores de resultado e para a compreensão de indicadores de processo envolvidos na avaliação da qualidade em serviços de saúde $e^{2,8-10}$.

A documentação analisada destaca aspectos estruturais à luz do referencial Donabediano e contribui para práticas inovadoras e humanizadas que primam pela difusão de cuidados baseados em evidência com ênfase na qualificação dos trabalhadores partícipes e na avaliação por meio de indicadores de qualidade desse processo em novos contextos ${ }^{1,7-10}$.

A capacitação em serviço foi demonstrada como um dos requisitos essenciais para desenvolvimento da gestão da qualidade em UTIN e demonstrou a necessidade de motivar e sensibilizar profissionais para o cuidado humanizado com RN como assistência efetiva e resolutiva ${ }^{2,5,6,8}$. A presente análise documental agrega, a esse corpo de conhecimento, a relevância sobre a utilização das metodologias ativas como potencializadoras para a incorporação dos sete pilares da qualidade propostos por Donabedian? .

A redução de taxas de infecção e sepse neonatal está associada com a implementação de capacitações direcionadas ao trabalho da equipe médica e de enfermagem, em especial com o foco da educação continuada baseada na realidade do trabalho, na melhoria da estrutura física, na valorização dos recursos humanos e na reorganização dos processos de trabalho ${ }^{17-19}$.

A supervisão da equipe coordenadora das oficinas de reedição e a avaliação da Comissão de Controle de Infecção Hospitalar evidenciaram, após 10 meses da capacitação em humanização, o aumento da rotatividade de 22 trabalhadores formados nas oficinas de reedição e, por conseguinte, constataram o incremento das taxas de infecção hospitalar na UTIN.

Esse efeito supramencionado, reforça as recomendações do Ministério da Saúde brasileiro ${ }^{14,15}$ sobre a capacitação das equipes de saúde como um dos planos de prevenção de infecção hospitalar. Entretanto, tais propostas requerem políticas institucionais e mudanças nos processos de trabalho, pois a alta rotatividade de profissionais dificulta o processo de educação em serviço pela redução do quadro de trabalhadores capacitados em caráter permanente ${ }^{17-19}$.

A defesa pela composição de uma equipe específica e qualificada contribui para a qualidade do cuidado, pois requer força de trabalho com saberes especializados ${ }^{3,6,17}$ e que enfatiza práticas de educação contínua baseada em estratégias de ensino baseadas em evidências que extrapolam as tendências pedagógicas tradicional e tecnicista para métodos de ensino e de aprendizagem humanistas, cognitivistas e críticos ${ }^{20}$.

\section{Considerações Finais}

Essa análise documental poderá subsidiar o delineamento de pesquisas observacionais (caso controle), de intervenção (quase experimental ou ensaio clínico) ou de avaliação (triangulação de métodos qualitativos, métodos mistos) em UTIN ou outros cenários promotores de cuidado com ênfase na gestão da qualidade e na educação permanente em saúde baseada em metodologias ativas.

Processos educativos com foco no profissional em seu contexto de trabalho e na resolução de problemas valorizam e aprimoram conhecimentos, habilidades e atitudes advindos de sua própria prática e do contexto institucional no qual estão inseridos. 
Ressalta-se que a compreensão dos processos educativos atrelados a políticas institucionais permite delinear estratégias alternativas para a redução da rotatividade das equipes treinadas a fim de garantir a segurança do RN e minimizar perdas técnicas, econômicas e administrativas e possíveis implicações ético-legais.

Com a divulgação dessa experiência pretende-se contribuir para a reflexão e o incentivo sobre o emprego de métodos ativos de ensino e de aprendizagem na educação permanente dos profissionais em hospitais de diferentes portes e em outras regiões do Brasil a fim de apreender abordagens e técnicas que resultam na melhoria contínua dos cuidados prestados e ajudam a consolidar os princípios da humanização da assistência.

\section{Referências Bibliográficas}

1. Opiyo N, English M. In-service training for health professionals to improve care of the seriously ill newborn or child in low and middle-income countries (Review). Cochrane Database Syst Rev. 2010;14(4). DOI: 10.1002/14651858.CD007071.pub2. 2. Rocha DKL, Ferreira HC. Estado da arte sobre o cuidar em neonatologia: compromisso da enfermagem com a humanização na unidade de terapia intensiva neonatal. Enfermagem em Foco. 2013;4(1):24-8.

3. Bittencourt WO, Lima ROS, Barbosa JLSB, Honório RB. Taxas de infecção hospitalar em uma unidade de terapia intensiva neonatal. Revista de Pesquisa: Cuidado é Fundamental Online. 2009;1(1):51-8. DOl: 10.9789/2175-5361.2009.v111.\%25p.

4. Ventura CMU, Alves JGB, Meneses JA. Eventos adversos em unidade de terapia intensiva neonatal. Rev Bras Enferm. 2012;65(1):49-55. DOI: 10.1590/S0034-71672012000100007.

5. Costa R, Padilha Ml. A unidade de terapia intensiva neonatal possibilitando novas práticas no cuidado ao recémnascido. Rev Gaúcha Enferm. 2011;32(2):248-55.

6. Reis LS, Silva EF, Waterkemper R, Lorenzini E, Cecchetto FH. Percepção da equipe de enfermagem sobre humanização em unidade de tratamento intensivo neonatal e pediátrica. Rev. Gaúcha Enferm. 2013;34(2):118-24. DOI: 10.1590/S198314472013000200015.

7. Donabedian A. The seven pillars of quality. Arch Pathol Lab Med. 1990:114(11):1115-8. 1997;121(11):1145-50.

8. Franco JN, Barros BPA, Vaidotas M, D'Innocenzo M. Nurses' perception on the results of quality indicators for the improvement of professional practice. Rev Bras Enferm. 2010;63(5):806-10. DOI: 10.1590/S0034-71672010000500018.

9. Pereira CM, Porto F. Quality indicators in neonatal intensive care: contributions in the management. Revista de Pesquisa: Cuidado é Fundamental Online. 2010;2(2):723-34. DOI: 10.9789/2175-5361.2010.v2i2.\%25p.

10. Vituri DW, Cacciari P, Gvozd R, Kuwabara CCT, Cardoso MGP. Indicadores de qualidade como estratégia para melhoria da qualidade do cuidado em um hospital universitário. Cienc Cuid Saúde. 2010;9(4):782-90. DOI: 10.4025/ cienccuidsaude.v9i4.13829.

11. Yin RK. Estudo de caso: planejamento e métodos. $4^{\mathrm{a}}$ ed. Porto Alegre: Bookman; 2010.

12. Bowling A. Research methods in health: investigating health and health services. $3^{a}$ ed. New York: Mc Graw Hill; 2010. 13. Miccas FL, Batista SHSS. Educação permanente em saúde: metassíntese. Rev. Saúde Pública. 2014;48(1):170-85. DOI: 10.1590/S0034-8910.2014048004498.

14. Brasil. Ministério da Saúde. Atenção humanizada ao recém-nascido de baixo peso: método canguru - caderno do tutor. Brasília; 2011.

15. Brasil. Ministério da Saúde. Atenção humanizada ao recém-nascido de baixo peso: método canguru. $2^{\mathrm{a}}$ ed. Brasília; 2009. 16.DonabedianA. Evaluating the quality of medical care. MilbankQ.2005;83(4):691-729. DOI: 10.1111/j.1468-0009.2005.00397.x. 17. Castro ECM, Leite AJM, Almeida MFB, Guinsburg R. Perinatal factors associated with early neonatal deaths in very low birth weight preterm infants in Northeast Brazil. BMC Pediatrics. 2014;14:312. DOI: 10.1186/s12887-014-0312-5.

18. Trotman H. Review of mortality of very low birthweight infants at the University Hospital of the West Indies over the past four decades. West Indian Med J. 2012;61(4):356-60.

19. Resende DS, Ó JM, Brito DD, Abdallah VOS, Gontijo Filho PP. Reduction of catheter-associated bloodstream infections through procedures in newborn babies admitted in a university hospital intensive care unit in Brazil. Rev Soc Bras Med Tropical. 2011;44(6):731-4.

20. Jobeth P. NICU Nurse educators: what evidence supports your teaching strategies? Neonatal Network. 2013;4:281-4. DOI: http://dx.doi.org/10.1891/0730-0832.32.4.281. 


\section{Alfredo Almeida Pina-Oliveira}

Endereço para correspondência - Av. Dr. Enéas de Carvalho Aguiar, $n^{\circ} 155,4^{\circ}$ andar, Bloco 6 , Bairro: Cerqueira César, CEP 05403-000, Cidade: São Paulo, SP, Brasil.

E-mail: aapo@ig.com.br

Lattes: http://lattes.cnpq.br/5159190517984235

Camila Aparecida Rodrigues Carriel - cacarriel@yahoo.com.br

Anna Maria Chiesa - amchiesa@usp.br

Enviado em 19 de setembro de 2014. Aceito em 27 de maio de 2015. 